Sharif University of Technology
Scientia Iranica
SCIENTIA
IRAN I CA
http://scientiairanica.sharif.edu

Invited Paper

\title{
Profile and wavefront optimization by metaheuristic algorithms for efficient finite element analysis
}

\author{
A. Kaveh ${ }^{\mathrm{a}, *}$ and Sh. Bijari ${ }^{\mathrm{b}}$ \\ a. Centre of Excellence for Fundamental Studies in Structural Engineering, Iran University of Science and Technology, Narmak, \\ Tehran, P.O. Box 16846-13114, Iran. \\ b. School of Civil Engineering, Iran University of Science and Technology, Narmak, Tehran, P.O. Box 16846-13114, Iran.
}

Received 25 December 2016; received in revised form 2 October 2017; accepted 28 January 2018

\author{
KEYWORDS \\ Profile and wavefront \\ reduction; \\ Ordering; \\ Colliding Bodies \\ Optimization $(\mathrm{CBO})$; \\ Enhanced Colliding \\ Bodies Optimization \\ (ECBO); \\ Tug of War \\ Optimization (TWO); \\ Vibrating Particles \\ System (VPS).
}

\begin{abstract}
To efficiently solve the equations that arise from finite element analysis, the stiffness matrix of a model should be structured. This can be achieved by reducing the profile or wavefront of the corresponding graph matrix of a structure depending on whether the skyline or frontal method is used. One of the efficient methods for achieving this goal is the method of King, extended by Sloan. In this paper, the coefficients of the priority function utilized in the generalized Sloan's method are optimized using the recently developed metaheuristic algorithm, called vibrating particles system. The results are compared with those of other metaheuristic algorithms consisting of the particle swarm optimization, colliding bodies optimization, enhanced colliding bodies optimization, and tug of war optimization. These metaheuristics are used for optimum nodal numbering of the graph models of the finite element meshes to reduce the profile and wavefront of the corresponding sparse matrices. The comparison of the results of the metaheuristic algorithms and those of the King and Sloan demonstrates the efficiency of the new metaheuristic algorithm utilized for profile and wavefront optimization.
\end{abstract}

(C) 2019 Sharif University of Technology. All rights reserved.

\section{Introduction}

The solution to the sparse systems of simultaneous equations comprises every main element of many problems in structural engineering. Such linear algebraic equations appear in the form of $A x=b$ arising from matrix structural analysis and finite element methods. These types of equations often involve a positive definite, symmetric, and sparse matrix coefficient $A$. For large structures, a great deal of computational time and memory is usually dedicated to finding a solution

\footnotetext{
*. Corresponding author. Tel.: +98 2144202710 ;

Fax: +9821 rra240398

E-mail address: alikaveh@iust.ac.ir (A. Kaveh)
}

to these equations. Therefore, some suitable specific patterns for the coefficients of the corresponding equations have been provided such as banded form, profile form, and blocked form. These patterns can often be obtained by the nodal ordering of the corresponding models.

In the Finite-Element Model (FEM) analysis, when the nodes have one degree of freedom, performing nodal ordering is equivalent to reordering the equations. In a more general problem, for each node with $m$ degree of freedom, there are $m$ coupled equations produced for each node. In such cases, re-sequencing is usually performed on the nodal numbering of the graph models to reduce the bandwidth, profile or wavefront, because the size of these problems is $m$ fold smaller than that with $m$ degree of freedom numbering. In this article, the mathematical model 
of the FEM is considered as an element clique graph, and nodal ordering is carried out to reduce the profile and wavefront of the corresponding matrices [1-3].

For nodal numbering in the solution of sparse systems, the following rule can be used: permuting rows and columns of a matrix by the proper renumbering of the nodes of the corresponding graph. Two important parameters in nodal ordering are profile and wavefront optimization. In fact, for sparse matrices, the size of the problem can be measured by the profile or wavefront of such matrices. These problems produced considerable interest in the past because of its practical relevance for a significant range of global optimization applications. Since the problem of nodal numbering is NP-Complete [4], many approximate approaches and heuristics have been proposed, examples of which can be found in Gibbs et al. [5], Cuthill and McKee [6], Kaveh [1], Bernardes and Oliveira [7], King [8], Kaveh and Behzadi [9], Kaveh and Roosta [10], and Koohestani and Poli [11].

Recently generated optimization methods consist of metaheuristic algorithms that solve complex problems. These techniques explore a feasible region based on both randomization and some specific rules through a group of search agents. The rules are usually inspired by the laws of natural phenomena [12].

As a recently developed type of metaheuristic approach, Colliding Bodies Optimization (CBO) was introduced and employed in structural problems by Kaveh and Mahdavi [13]. CBO is a multi-agent method and is inspired by a one-dimensional collision between two agents. Each object is considered as a body with a specific mass and a velocity. A collision occurs between a pair of bodies, and the new positions of the colliding bodies are updated based on the laws of collision. The enhanced colliding bodies optimization introduced by Kaveh and Ilchi Ghazaan [14] employs memory in order to save the best-so-far position to improve the performance of $\mathrm{CBO}$ without increasing the computer execution time. This algorithm also utilizes a mechanism to escape local optima. A multiagent metaheuristic algorithm, called tug of war optimization, was introduced by Kaveh and Zolghadr [15]. This technique models each candidate solution as a team engaged in a series of tug of war competitions. The weight of the teams is determined based on the quality of the corresponding solutions, and the value of pulling force that a team can exert on the rope is assumed to be proportional to its weight. VPS is a population-based optimization algorithm, which is inspired by a free vibration of a single degree of freedom systems with viscous damping, as was introduced by Kaveh and Ilchi Ghazaan [16]. In this method, the solution candidates are considered as agents that gradually approach their equilibrium positions. In order to ensure a proper balance between exploration and exploitation, equilibrium positions are attained from the current population and the best historical position.

The rest of this paper is organized as follows: in Section 2, some definitions of graph theory, profile, and wavefront are presented. CBO, ECBO, TWO, and VPS algorithms are briefly stated in Section 3. In order to show the performance of these methods in reducing the profile and wavefront of the stiffness matrix, eight examples are presented in Section 4. The last section concludes the present study.

\section{Problem definition}

\subsection{Definitions from the theory of graphs}

Let $G(N, M)$ be a graph with a set of members $M(|M|=m)$ and a set of nodes $N(|N|=n)$ together with a relation of incidence. The degree of a node is the number of members, which are incidental to that node; 1-weighted degree of a node is defined as the sum of the degrees of its adjacent nodes. A spanning tree is a tree containing all the nodes of $S$. The Shortest Route Tree $\left(\mathrm{SRT}_{n_{0}}\right)$ rooted in a considered node (starting node), $n_{0}$, is a spanning tree where the distance between every node $n_{j}$ of $S$ and $n_{0}$ is minimum and where the distance between the two nodes is taken to be the number of members in the shortest path between these nodes. A contour $C_{k}^{n_{0}}$ contains all the nodes with equal distance $k$ from node $n_{0}$. The number of contours of an $\mathrm{SRT}_{n_{0}}$ is known as its depth, denoted by $d\left(\mathrm{SRT}_{n_{0}}\right)$, and the highest number of nodes in a contour shows the width of $\mathrm{SRT}_{n_{0}}$. A label $A s$ of $G$ assigns a set of integers $\{1,2,3, \cdots, n\}$ to the nodes of graph $G$. As $(i)$ is the label or the integer assigned to node $i$, and each node has a different label [2].

The profile of an $N \times N$ matrix related to graph $G$, for the assignment process by $A s(i)$, is defined as follows:

$$
P_{A s}=\sum_{i=1}^{N} b_{i}
$$

where the row bandwidth, $b_{i}$, for row $i$ is defined as the number of inclusive entries from the first nonzero element in the row to the $(i+1)$ th entry for this assignment. The efficiency of the given ordering for the profile solution scheme corresponds to the number of active equations during each step of the factorization process. Formally, row $j$ is defined to be active during elimination of column $i$ if $j \geq i$, and there exists $a_{i k}=0$ with $k \leq i$. Hence, in the $i$ th stage of the factorization, the number of active equations is the number of rows of profile that intersect in column $i$, where the already eliminated rows are ignored. Let $f_{i}$ denote the number of equations that are active during the elimination of variable $x_{i}$. It follows from the symmetric structures 
of the matrix that:

$$
P_{A s}=\sum_{i=1}^{N} f_{i}=\sum_{i=1}^{N} b_{i}
$$

where $f_{i}$ is the frontwidth or wavefront. Assuming that $N$ and the average value of $f_{i}$ are considerably large, it can be shown that a complete profile or front factorization needs approximately $O\left(N F_{r m s}^{2}\right)$ operations, where $F_{r m s}$ is the root-mean square frontwidth, which is defined as follows:

$$
F_{r m s}=\left[\frac{1}{N} \sum_{i=1}^{N} f_{i}^{2}\right]^{0.5}
$$

For the purpose of obtaining an optimal nodal ordering in the profile and frontwidth reduction problems, the set of integers $\{1,2,3, \cdots, n\}$ should be assigned to the nodes of $G$ using a priority function, and the coefficients of the priority functions are obtained using PSO, CBO, ECBO, TWO, and VPS algorithms.

\subsection{An algorithm based on priority queue for profile and wavefront reduction}

The nodal numbering in a priority queue is carried out through the assignment of status based on the numbering approach of King [8]. King's method was generalized by Sloan [17] by introducing a priority queue that controls the order to be followed in the numbering of the nodes. This algorithm comprises two phases:

Phase 1: Selecting a pair of pseudo-peripheral (pseudo-diameter) nodes;

Phase 2: Nodal numbering.

In Phase 1, a pair of nodes is selected as starting and ending nodes according to the following steps:

Step 1: Choose an arbitrary node $s$ of minimum degree;

Step 2: Generate the shortest route tree $\mathrm{SRT}_{s}=$ $\left\{C_{1}^{s}, C_{2}^{s}, \cdots, C_{d}^{s}\right\}$ rooted in $s$. Let $S$ be the list of the nodes of $C_{d}^{s}$, which is stored in the order of increasing degree;

Step 3: Decompose $S$ into subsets $S_{j}$ of cardinality $\left|S_{j}\right|, j=1,2, \cdots, \Delta$, where $\Delta$ is the maximum degree of any node of $S$ such that all nodes of $S_{j}$ are characterized by degree $j$. Generate an SRT from each node $y$ of $S$ for the first $1 \leq m_{j} \leq \Delta$. If $d\left(\mathrm{SRT}_{y}\right)>d\left(\mathrm{SRT}_{s}\right)$, then set $s=y$ and go to Step 2;

Step 4: Let $e$ be the root of the longest SRT that has the smallest width. When the algorithm terminates, $s$ and $e$ are the end points of a pseudo-diameter.

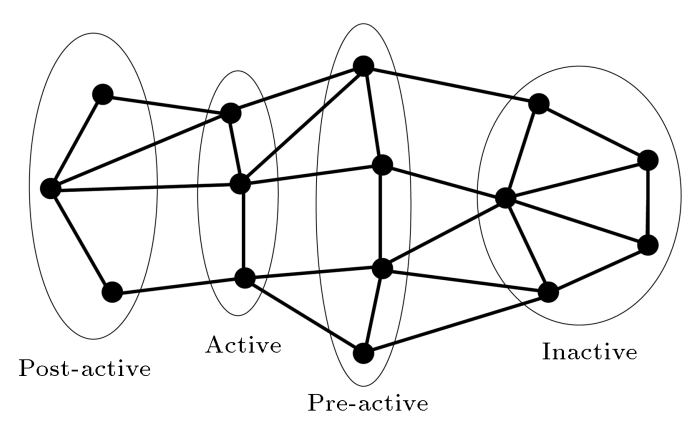

Figure 1. Different statuses of nodes.

In Phase 2 the nodes of an element clique graph are reordered, and it is ensured that the position of a node in this reordering phase follows a priority rule according to the following steps:

Step 1: Find the status of all nodes. A node can be found in one status of the following cases, as shown in Figure 1. A node that has been assigned a new label is considered as post-active. Nodes that are adjacent to a post-active node, yet do not have a post-active status, are called active. Each node which is adjacent to an active node, but is not post-active or active, is said to be pre-active. The nodes which are not postactive, active, or pre-active are considered as inactive;

Step 2: Prepare a list of the candidate nodes, consisting of active and pre-active nodes, for labeling in the next step;

Step 3: Calculate the priority number of all the candidate nodes. For node $i$, the number is obtained from the following relationship:

$$
P_{i}=W_{1} \times \delta_{i}-W_{2} \times D_{i}
$$

where $W_{1}$ and $W_{2}$ are integer weights (suggested as $W_{1}=1$ and $W_{2}=2$ in the original Sloan's algorithm), $\delta_{i}$ is the distance between each node $i$ and the end node, and $D_{i}$ is the incremental degree of node $i$, which is defined as follows:

$$
D_{i}=d_{i}-c_{i}+k_{i}
$$

where $d_{i}$ is the degree of node $i, c_{i}$ is the number of active and post-active nodes adjacent to node $i$, and $k_{i}$ is zero if node $i$ is active or post-active, and unity otherwise;

Step 4: Select the node with the highest priority among the candidate nodes and label it;

Step 5: Repeat Steps 1-4 until all the nodes of the graph are labeled.

In Eq. (4) if $W_{1}=0$ and $W_{2}=1$, the node labeling algorithm becomes identical to the one proposed by King [8]. 


\subsection{The priority function with new integer weights}

According to Eq. (4), a linear priority function of two graph parameters is employed in Sloan's algorithm, and the weights determine the rank of each parameter. Sloan has recommended the pair $W_{1}=1$ and $W_{2}=2$ for the weights. However, some research results show that, for some problems, using other values leads to better results.

The priority was generalized by Kaveh and Rahimi Bondarabdy [18] to a linear function of vectors of graph parameters as follows:

$$
F_{i}=\sum_{i=1}^{L} W_{i} \times G_{i},
$$

where $G_{i}(i=1,2, \cdots, L)$ are the normalized Ritz vectors representing the graph parameters, $W_{i}(i=$ $1,2, \cdots, L)$ are the weights of the Ritz vectors (Ritz coordinates), which are unknown. That is, one can utilize $L$ characteristics of a graph to define the priority function and find the weights that can guide the algorithm to choose an optimal profile or wavefront.

$L=2$ characteristics of the graph model are employed in Sloan's algorithm. Here, we find the best sets of weights for the priority function with $L=2$ and 5 . These sets of integer weights are found by utilizing PSO, CBO, ECBO, TWO, and VPS algorithms.

The method of $L=2$ is presented as the first case. The vectors of graph properties are taken similar to those of Sloan's strategy. In the second case, the method of $L=5$, which uses five vectors $G_{i}(i=$ $1,2, \cdots, 5)$, is presented as follows:

- $G_{1}$ : Degrees of the nodes;

- $G_{2}$ : The node distance from the end node;

- $G_{3}$ : The node distance from the starting node;

- $G_{4}$ : The 1-weighted degree;

- $G_{5}$ : The width of an SRT rooted in the starting node.
Once the vectors of graph parameters are formed, their weights can be obtained using PSO, CBO, ECBO, TWO, and VPS algorithms.

\section{The utilized metaheuristic algorithms}

Metaheuristic algorithms have been previously utilized to optimize the coefficients of the generalized Sloan function. Genetic algorithm [19], ant colony [20], charged system search [21], CBO, ECBO, and tug of war [22] are some of these applications. In this paper, VPS is added to the 3 algorithms used in the latter reference, and a more extensive comparison is made.

In this section, a brief description and pseudo codes of the colliding bodies optimization algorithm, its enhanced version, and then two new algorithms, called tug of War Optimization algorithm and vibrating particles system algorithm, are presented and employed to calculate the optimum values of the coefficients.

\subsection{Colliding bodies optimization}

The collision between two objects is a natural phenomenon. The colliding bodies optimization technique was developed based on this occurrence by Kaveh and Mahdavi [13]. In this technique, one object collides with another and, thus, they move towards a minimum energy level. CBO utilizes a simple formulation, does not employ memory for saving the best-so-far solutions, and requires no internal parameter. For further details, reader mays refer to [13]. The pseudo code of CBO is provided in Figure 2.

\subsection{Enhanced colliding bodies optimization}

Enhanced colliding bodies optimization is a modified version of CBO, which improves CBO to achieve highly reliable solutions, as was introduced by Kaveh and Ilchi Ghazaan [14]. The introduction of memory increases the convergence speed of ECBO more than that of CBO. Furthermore, changing some components of CBO helps ECBO escape local optima. For further

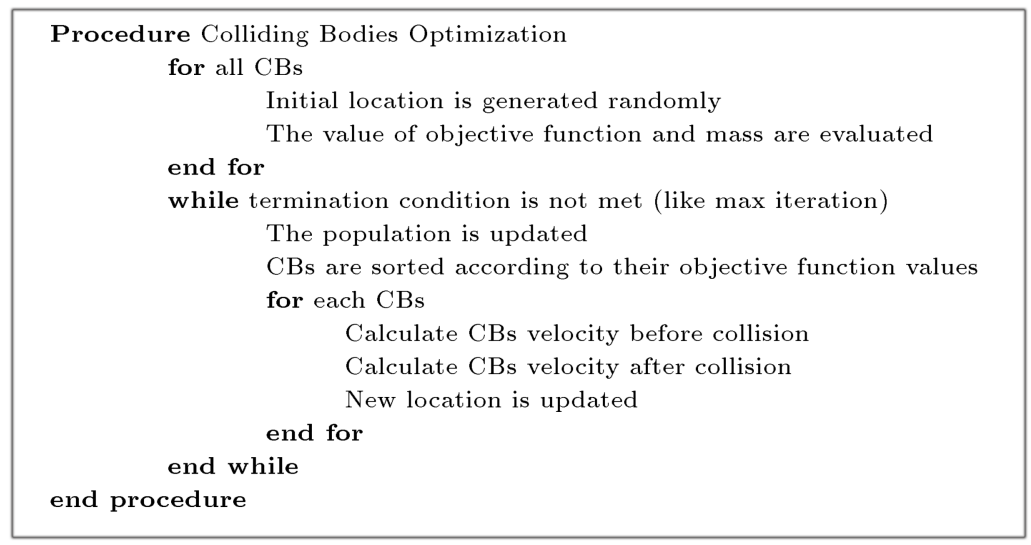

Figure 2. Pseudo code of the colliding bodies optimization algorithm. 




Figure 3. Pseudo code of the enhanced colliding bodies optimization algorithm.

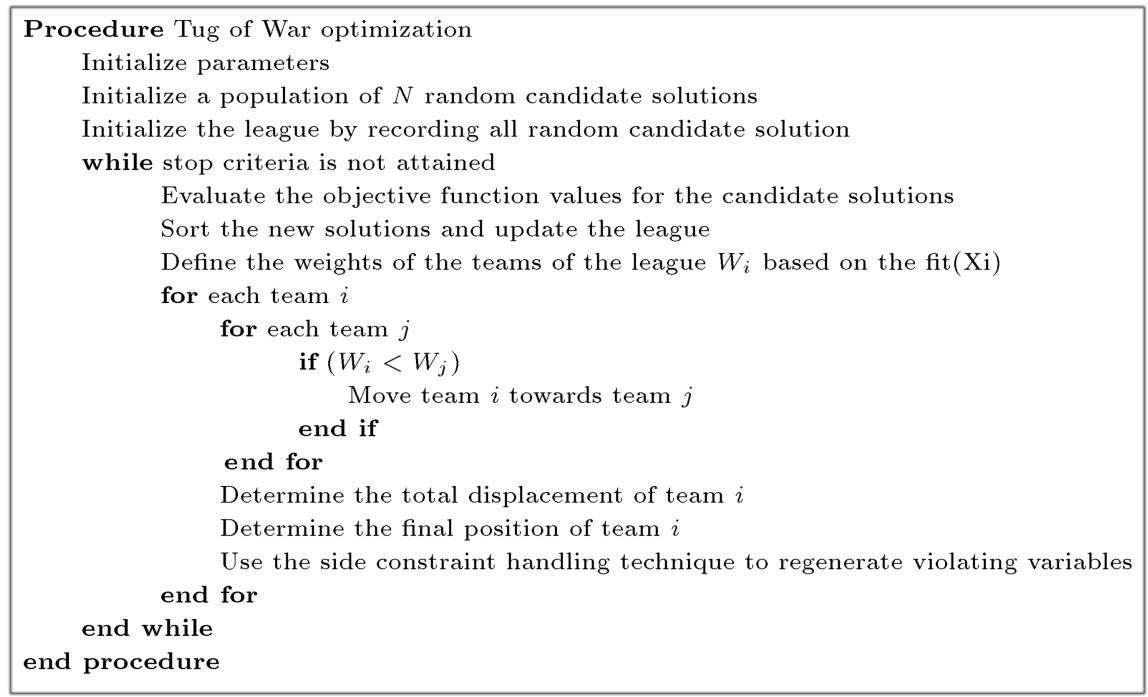

Figure 4. Pseudo code of the tug of war optimization algorithm.

details, readers may refer to [14]. The pseudo code of ECBO is provided in Figure 3.

\subsection{Tug of War Optimization (TWO) algorithm}

TWO is a population-based metaheuristic algorithm, which was introduced by Kaveh and Zolghadr [15]. This approach models each candidate solution $X_{i}=$ $\left\{x_{i, j}\right\}$ as a team engaged in a series of tug of war competitions. The weight of the teams is determined based on the quality of the corresponding solutions, and the value of pulling force which a team can exert on the rope is assumed to be proportional to its weight. Naturally, the opposite team will have to maintain at least the same value of force in order to sustain its grip of the rope. The lighter team accelerates toward the heavier team, and this forms the convergence operator of TWO method. The method improves the quality of the solutions iteratively by keeping a proper exploration/exploitation balance and employing the described convergence operator. The pseudo code of TWO is provided in Figure 4.

\subsection{Vibrating particles system algorithm}

The vibrating particles system is a metaheuristic method and is inspired by the damped free vibration of single degree of freedom systems, as was introduced by Kaveh and Ilchi Ghazaan [16]. This approach involves a number of candidate solutions, representing the particles system. The particles are initialized randomly in an $n$-dimensional search space and gradually approach their equilibrium positions. To keep the balance between the exploration and exploitation, these equilibrium positions are achieved through the 


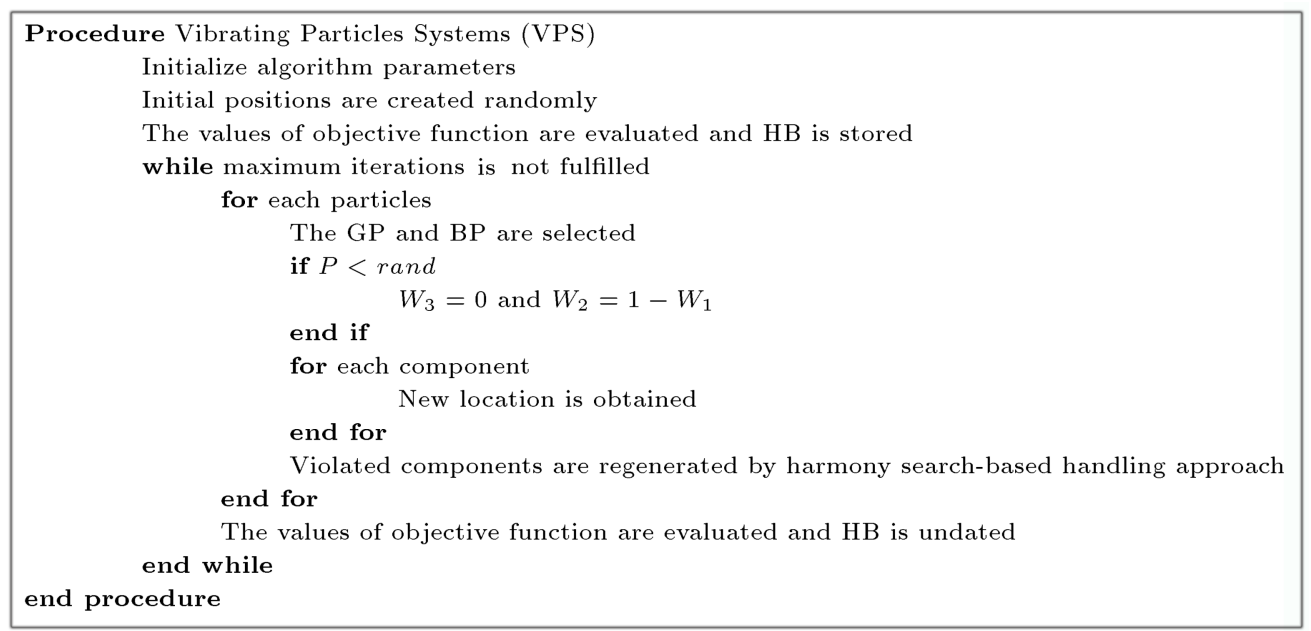

Figure 5. Pseudo code of the vibrating particles system algorithm.

current population and the historically best position. The pseudo code of VPS is provided in Figure 5.

\section{Numerical examples}

In this section, eight Finite Element Meshes (FEMs) are considered. Element clique graph is a type of graph model that is employed for transferring topological properties of finite element models into connectivity properties of graphs [3]. The nodes of this graph model are the same as those of the corresponding finite element model with the nodes of each element being cliqued, and the multiple members in the entire graph are replaced by single members. Ten problems are presented to show the performance and efficiency of some new optimization algorithms. Four test problems are presented by Everstine [23]. The well-known standard PSO algorithm, two new algorithms, namely the colliding bodies optimization and enhanced colliding bodies optimization, and two recently developed methods called tug of war optimization and vibrating particles system are applied to reduce the profile and wavefront. The results of the reduction of profile and wavefront with $L=2$ and 5 methods are compared with those of the Sloan and King's algorithms in Tables 1 to 16 , respectively.

Example 1. A tower with 87 nodes and 227 elements is considered. The finite element mesh of this model is shown in Figure 6 . The efficiency of the abovementioned algorithms is tested by this model for profile and wavefront reduction problems. The obtained results are given in Tables 1 and 2, respectively. The quality of the results is provided in these tables.

Example 2. The schematic of a finite element model of power supply housing is presented in Figure 7. This

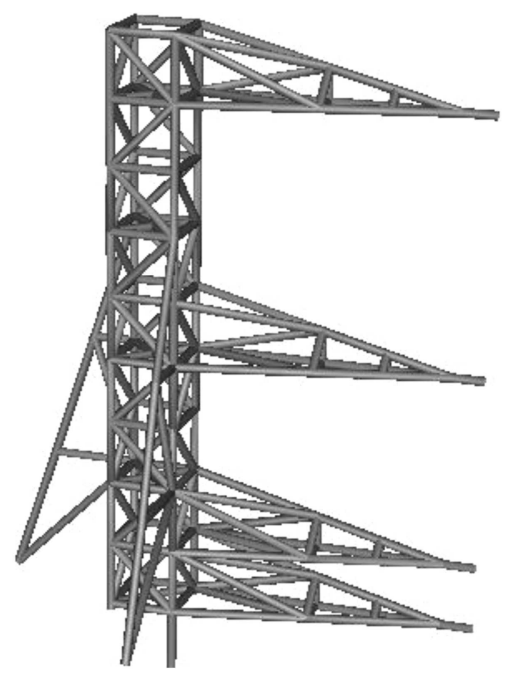

Figure 6. Schematic of the FEM of a tower.



Figure 7. Schematic of the FEM of power supply housing.

model has 307 nodes and 1108 elements. The results of profile and wavefront reduction problems by employing optimization algorithms are given in Tables 3 and 4 , respectively. 
Table 1. Comparison of the results of profile reduction for the tower problem.

\begin{tabular}{|c|c|c|c|c|c|c|c|c|}
\hline & Algorithm & No. of vectors & $W_{1}$ & $W_{2}$ & $W_{3}$ & $W_{4}$ & $W_{5}$ & Profile \\
\hline \multirow{12}{*}{ 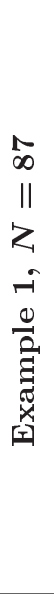 } & Sloan & & 1 & 2 & & & & 778 \\
\hline & King & & 0 & 1 & & & & 795 \\
\hline & PSO & $L=2$ & 0.1036 & 0.3706 & & & & 767 \\
\hline & & $L=5$ & -0.0144 & -0.6110 & 0.7313 & -0.0006 & 0.2632 & 571 \\
\hline & $\mathrm{CBO}$ & $L=2$ & 0.2719 & 0.0641 & & & & 692 \\
\hline & & $L=5$ & -0.0589 & -0.8641 & 0.9263 & -0.0009 & 0.8139 & 571 \\
\hline & $\mathrm{ECBO}$ & $L=2$ & 0.2830 & 0.8495 & & & & 767 \\
\hline & & $L=5$ & 0.0217 & -0.8665 & 0.9936 & -0.0001 & -0.0597 & 571 \\
\hline & TWO & $L=2$ & 0.2707 & 0.9853 & & & & 767 \\
\hline & & $L=5$ & 0.0011 & -0.4868 & 0.5443 & -0.0015 & -0.9865 & 571 \\
\hline & VPS & $L=2$ & 0.0640 & 0.2222 & & & & 767 \\
\hline & & $L=5$ & -0.0175 & -0.7657 & 0.8836 & -0.0001 & 0.8663 & 571 \\
\hline
\end{tabular}

Table 2. Comparison of the results of wavefront reduction for the tower problem.

\begin{tabular}{|c|c|c|c|c|c|c|c|c|}
\hline & Algorithm & No. of vectors & $W_{1}$ & $W_{2}$ & $W_{3}$ & $W_{4}$ & $W_{5}$ & $F_{r m s}$ \\
\hline \multirow{12}{*}{ 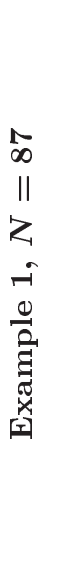 } & Sloan & & 1 & 2 & & & & 8.7382 \\
\hline & King & & 0 & 1 & & & & 9.2189 \\
\hline & PSO & $L=2$ & 0.2007 & 0.6255 & & & & 9.6900 \\
\hline & & $L=5$ & 0.0009 & -0.5316 & 0.5742 & -0.0009 & 0.5547 & 6.9124 \\
\hline & $\mathrm{CBO}$ & $L=2$ & 0.2875 & 0.8730 & & & & 9.6900 \\
\hline & & $L=5$ & 0.0004 & 0.9800 & -0.9047 & -0.0008 & 0.3827 & 6.9124 \\
\hline & $\mathrm{ECBO}$ & $L=2$ & 0.2428 & 0.8169 & & & & 9.6900 \\
\hline & & $L=5$ & 0.0005 & -0.8408 & 0.9966 & -0.0027 & 0.1399 & 6.9124 \\
\hline & TWO & $L=2$ & 0.2727 & 0.9664 & & & & 9.6900 \\
\hline & & $L=5$ & -0.1069 & -0.6872 & 0.6581 & -0.0040 & 0.8902 & 7.3085 \\
\hline & VPS & $L=2$ & 0.0694 & 0.2674 & & & & 9.6900 \\
\hline & & $L=5$ & 0.0000 & -0.7642 & 0.9004 & -0.0022 & 0.0390 & 6.9124 \\
\hline
\end{tabular}

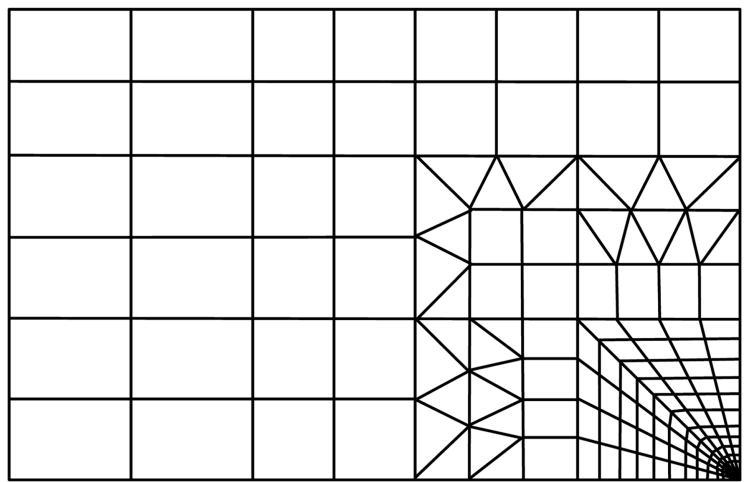

Figure 8. Schematic of the hull w/refinement model.

Example 3. A hull w/refinement model containing 310 nodes and 1069 elements is considered in Figure 8. Tables 5 and 6 provide the values of the profile and wavefront reduction, respectively, and the results can be compared with those of these tables.

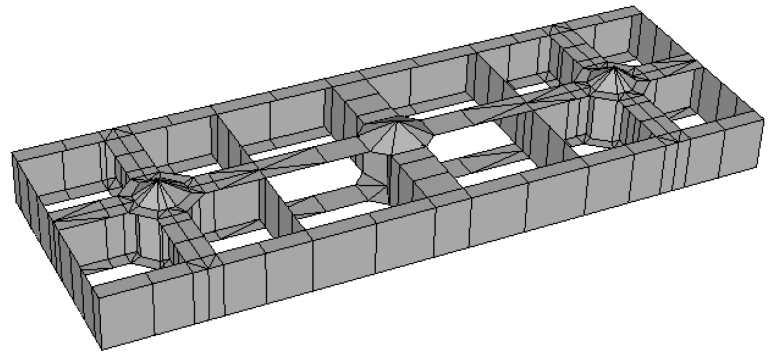

Figure 9. FEM of a barge.

Example 4. The FEM of a barge with 419 nodes and 1579 elements is considered, as shown in Figure 9. Tables 7 and 8 provide the comparison results of profile and wavefront reduction problems by utilizing PSO, CBO, ECBO, TWO, and VPS algorithms for this example.

Example 5. A Z-shaped finite element model of a 
Table 3. Comparison of the results of profile reduction for the power supply housing problem.

\begin{tabular}{|c|c|c|c|c|c|c|c|c|}
\hline & Algorithm & No. of vectors & $W_{1}$ & $W_{2}$ & $W_{3}$ & $W_{4}$ & $W_{5}$ & Profile \\
\hline \multirow{12}{*}{  } & Sloan & & 1 & 2 & & & & 8730 \\
\hline & King & & 0 & 1 & & & & 9095 \\
\hline & PSO & $L=2$ & 0.0042 & 0.2228 & & & & 8205 \\
\hline & & $L=5$ & -0.0742 & 0.6049 & -0.0674 & 0.0103 & -0.5000 & 8421 \\
\hline & $\mathrm{CBO}$ & $L=2$ & 0.0850 & 0.8204 & & & & 8148 \\
\hline & & $L=5$ & 0.0174 & -0.2591 & 0.9308 & -0.0035 & 0.1450 & 8262 \\
\hline & $\mathrm{ECBO}$ & $L=2$ & 0.0546 & 0.9436 & & & & 8205 \\
\hline & & $L=5$ & -0.0035 & -0.0601 & 0.3642 & -0.0010 & -0.9357 & 8262 \\
\hline & TWO & $L=2$ & 0.0248 & 0.3105 & & & & 8189 \\
\hline & & $L=5$ & -0.5751 & -0.8167 & -0.1357 & -0.5099 & 0.6424 & 8004 \\
\hline & VPS & $L=2$ & 0.0168 & 0.2476 & & & & 8205 \\
\hline & & $L=5$ & 0.0009 & -0.1628 & 0.9542 & -0.0002 & 0.7594 & 8213 \\
\hline
\end{tabular}

Table 4. Comparison of the results of wavefront reduction for the power supply housing problem.

\begin{tabular}{|c|c|c|c|c|c|c|c|c|}
\hline & Algorithm & No. of vectors & $W_{1}$ & $W_{2}$ & $W_{3}$ & $W_{4}$ & $W_{5}$ & $F_{r m s}$ \\
\hline \multirow{12}{*}{ 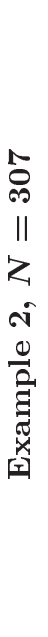 } & Sloan & & 1 & 2 & & & & 31.2292 \\
\hline & King & & 0 & 1 & & & & 32.0931 \\
\hline & PSO & $L=2$ & 0.1578 & 0.9402 & & & & 27.5724 \\
\hline & & $L=5$ & 0.0105 & 0.1509 & -0.9033 & -0.0056 & -0.6496 & 30.5202 \\
\hline & $\mathrm{CBO}$ & $L=2$ & 0.1641 & 0.7762 & & & & 30.6501 \\
\hline & & $L=5$ & -0.0375 & -0.1102 & 0.9901 & 0.0056 & -0.0212 & 28.426 \\
\hline & $\mathrm{ECBO}$ & $L=2$ & 0.0777 & 0.9292 & & & & 27.6011 \\
\hline & & $L=5$ & -0.0416 & -0.0428 & 0.9934 & 0.0080 & -0.0424 & 28.4111 \\
\hline & TWO & $L=2$ & 0.0069 & 0.0459 & & & & 27.2907 \\
\hline & & $L=5$ & -0.3605 & -0.9187 & -0.0700 & -0.7603 & -0.7260 & 27.2771 \\
\hline & VPS & $L=2$ & 0.0368 & 0.7499 & & & & 27.2907 \\
\hline & & $L=5$ & 0.0042 & -0.0490 & 0.8914 & -0.0011 & -0.3126 & 27.7194 \\
\hline
\end{tabular}

Table 5. Comparison of the results of profile reduction for the hull w/refinement model.

\begin{tabular}{|c|c|c|c|c|c|c|c|c|}
\hline & Algorithm & No. of vectors & $W_{1}$ & $W_{2}$ & $W_{\mathbf{3}}$ & $W_{4}$ & $W_{5}$ & Profile \\
\hline \multirow{12}{*}{  } & Sloan & & 1 & 2 & & & & 3211 \\
\hline & King & & 0 & 1 & & & & 3222 \\
\hline & PSO & $L=2$ & 0.4576 & 0.1340 & & & & 3171 \\
\hline & & $L=5$ & -0.0317 & 0.5209 & -0.3798 & -0.0137 & 0.9275 & 3170 \\
\hline & $\mathrm{CBO}$ & $L=2$ & 0.9282 & 0.2813 & & & & 3171 \\
\hline & & $L=5$ & 0.0290 & 0.3456 & 0.9489 & -0.0134 & 0.3580 & 3125 \\
\hline & $\mathrm{ECBO}$ & $L=2$ & 0.9380 & 0.2136 & & & & 3179 \\
\hline & & $L=5$ & -0.0108 & -0.1277 & 0.8426 & -0.0031 & 0.1786 & 3125 \\
\hline & TWO & $L=2$ & 0.9556 & 0.3112 & & & & 3179 \\
\hline & & $L=5$ & 0.4208 & -0.9040 & -0.8360 & -0.9611 & 0.6841 & 2979 \\
\hline & VPS & $L=2$ & 0.1361 & 0.0405 & & & & 3171 \\
\hline & & $L=5$ & -0.0368 & -0.1685 & 0.9088 & -0.0012 & -0.8294 & 3125 \\
\hline
\end{tabular}


Table 6. Comparison of the results of wavefront reduction for the refined finite element model.

\begin{tabular}{|c|c|c|c|c|c|c|c|c|}
\hline & Algorithm & No. of vectors & $W_{1}$ & $W_{2}$ & $W_{3}$ & $W_{4}$ & $W_{5}$ & $F_{r m s}$ \\
\hline \multirow{12}{*}{ 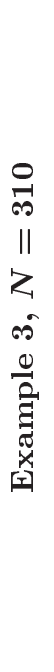 } & Sloan & & 1 & 2 & & & & 10.5232 \\
\hline & King & & 0 & 1 & & & & 10.5841 \\
\hline & PSO & $L=2$ & 0.4899 & 0.1379 & & & & 10.4361 \\
\hline & & $L=5$ & 0.0326 & -0.1466 & 0.7705 & -0.0080 & 0.2158 & 10.2531 \\
\hline & $\mathrm{CBO}$ & $L=2$ & 0.8400 & 0.2420 & & & & 10.4108 \\
\hline & & $L=5$ & 0.0115 & -0.0681 & 0.4104 & -0.0024 & 0.0422 & 10.2716 \\
\hline & $\mathrm{ECBO}$ & $L=2$ & 0.8420 & 0.8420 & & & & 10.4108 \\
\hline & & $L=5$ & -0.0502 & -0.1924 & 0.8743 & -0.0003 & 0.4107 & 10.2716 \\
\hline & TWO & $L=2$ & 0.9624 & 0.2722 & & & & 10.4108 \\
\hline & & $L=5$ & -0.7532 & -0.6871 & -0.6053 & -0.9134 & -0.0993 & 9.7714 \\
\hline & VPS & $L=2$ & 0.5418 & 0.1575 & & & & 10.4361 \\
\hline & & $L=5$ & -0.0307 & -0.1404 & 0.8417 & -0.0008 & 0.2558 & 10.2716 \\
\hline
\end{tabular}

Table 7. Comparison of the results of profile reduction for the barge problem.

\begin{tabular}{|c|c|c|c|c|c|c|c|c|}
\hline & Algorithm & No. of vectors & $W_{1}$ & $W_{2}$ & $W_{3}$ & $W_{4}$ & $W_{5}$ & Profile \\
\hline \multirow{12}{*}{  } & Sloan & & 1 & 2 & & & & 9113 \\
\hline & King & & 0 & 1 & & & & 9301 \\
\hline & PSO & $L=2$ & 0.5179 & 0.0881 & & & & 8808 \\
\hline & & $L=5$ & -0.0932 & -0.2695 & 0.7979 & 0.0075 & 0.4298 & 8651 \\
\hline & $\mathrm{CBO}$ & $L=2$ & 0.7913 & 0.1417 & & & & 8808 \\
\hline & & $L=5$ & -0.1436 & -0.2777 & 0.9433 & 0.0140 & 0.2705 & 8646 \\
\hline & $\mathrm{ECBO}$ & $L=2$ & 0.8527 & 0.1544 & & & & 8802 \\
\hline & & $L=5$ & -0.9124 & 0.4662 & -0.7636 & -0.0054 & -0.0497 & 8640 \\
\hline & TWO & $L=2$ & 0.9766 & 0.1637 & & & & 8804 \\
\hline & & $L=5$ & 0.0331 & 0.9569 & -0.1261 & -0.0064 & 0.9825 & 8643 \\
\hline & VPS & $L=2$ & 0.3376 & 0.0664 & & & & 8804 \\
\hline & & $L=5$ & 0.0287 & -0.2435 & 0.9158 & -0.0092 & -0.1923 & 8630 \\
\hline
\end{tabular}

Table 8. Comparison of the results of wavefront reduction for the barge problem.

\begin{tabular}{|c|c|c|c|c|c|c|c|c|}
\hline & Algorithm & No. of vectors & $W_{1}$ & $W_{2}$ & $W_{3}$ & $W_{4}$ & $W_{5}$ & $F_{r m s}$ \\
\hline \multirow{12}{*}{ 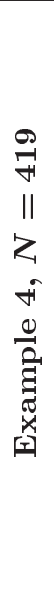 } & Sloan & & 1 & 2 & & & & 22.5524 \\
\hline & King & & 0 & 1 & & & & 23.0926 \\
\hline & PSO & $L=2$ & 0.7145 & 0.1413 & & & & 21.6733 \\
\hline & & $L=5$ & 0.1294 & 0.7287 & -0.2671 & -0.0203 & -0.2336 & 21.3466 \\
\hline & $\mathrm{CBO}$ & $L=2$ & 0.9711 & 0.1722 & & & & 21.6906 \\
\hline & & $L=5$ & -0.0756 & 0.8504 & -0.2259 & 0.0063 & -0.6770 & 21.2456 \\
\hline & $\mathrm{ECBO}$ & $L=2$ & 0.2526 & 0.0449 & & & & 21.6583 \\
\hline & & $L=5$ & -0.0531 & -0.2415 & 0.9790 & 0.0044 & -0.0757 & 21.2783 \\
\hline & TWO & $L=2$ & 0.4787 & 0.0870 & & & & 21.6733 \\
\hline & & $L=5$ & -0.0402 & 0.9657 & -0.3727 & 0.0033 & 0.8098 & 21.2683 \\
\hline & VPS & $L=2$ & 0.3065 & 0.0611 & & & & 21.6593 \\
\hline & & $L=5$ & -0.1294 & 0.9687 & -0.3533 & 0.0105 & -0.7296 & 21.2456 \\
\hline
\end{tabular}




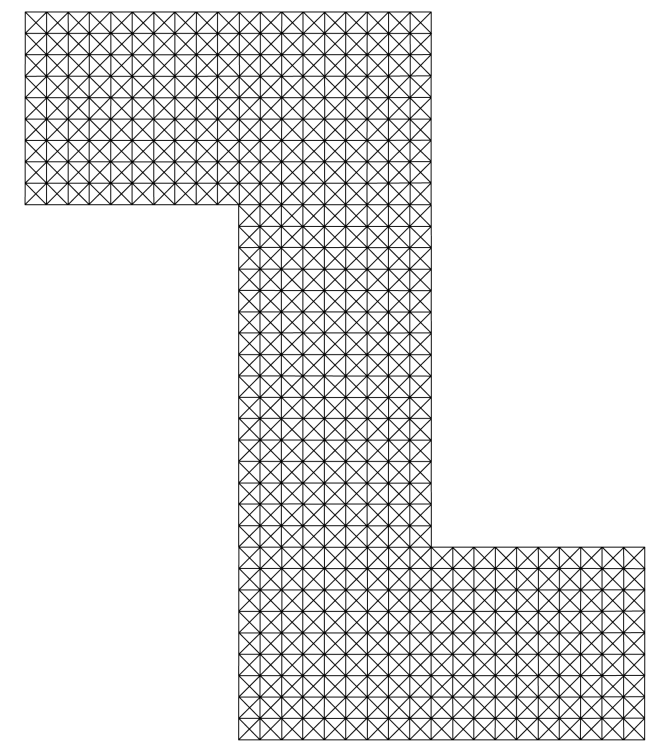

Figure 10. FEM of a shear wall.

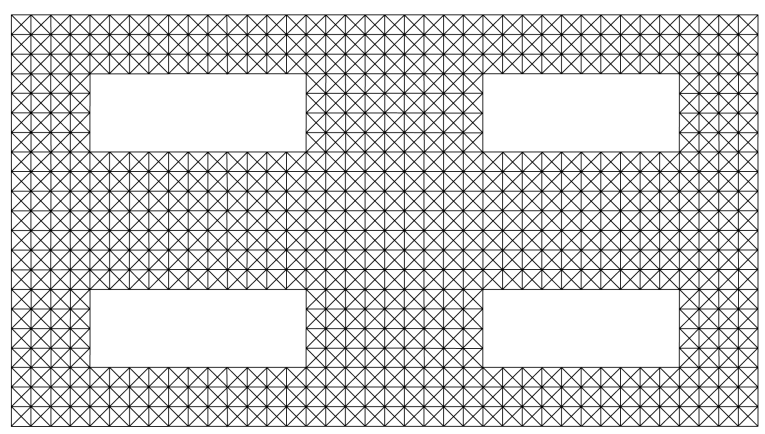

Figure 11. The element clique graph of a rectangular FEM with four openings.

shear wall with 550 nodes is considered. The element clique graph of this model is shown in Figure 10. The performance of the algorithms is tested by this model for profile and wavefront reduction problems. The results are given in Tables 9 and 10, respectively.

Example 6. The element clique graph of a rectangular FEM with four openings, as shown in Figure 11, contains 760 nodes. The performances of PSO, CBO, ECBO, TWO, and VPS algorithms are tested by this model for profile and wavefront reduction problems. The results for these problems are represented in Tables 11 and 12, respectively.

Example 7. The grid model of a fan with $1 \mathrm{D}$ beam elements containing 1575 nodes is considered, as shown in Figure 12. Similar to the previous examples, the obtained values by the algorithms for profile and wavefront reduction problems are given in Tables 13 and 14 , respectively, where the results can easily be compared.

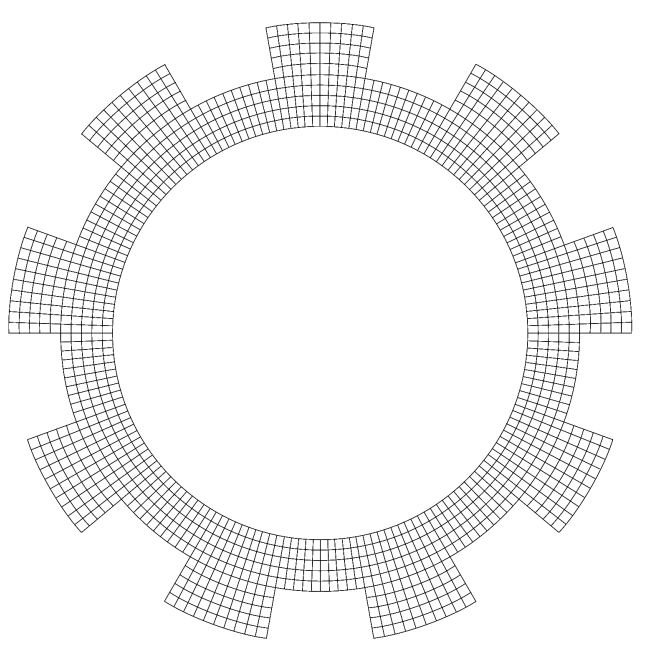

Figure 12. The graph model of a fan.



Figure 13. Finite element grid model of a shear wall.

Example 8. The graph model of an H-shaped shear wall with 4949 nodes is considered, as shown in Figure 13. The performance of the above-mentioned algorithms is verified by this model, and the results of these algorithms for profile and wavefront reduction problems are provided in Tables 15 and 16, respectively.

\section{Concluding remarks}

The main aim of this study is to use VPS algorithm for profile and wavefront reduction of finite element mesh matrices for the first time and to present the pseudo codes for CBO, ECBO, TWO, and VPS algorithms. The results of these algorithms, when applied to 8 real-life finite element models, were then compared. According to Tables 1 to 16, it can be observed that the achieved results of these algorithms were quite satisfactory, compared to the well-known graph theoretical method of the King and Sloan. 
Table 9. Comparison of the results of profile reduction for the shear wall problem.

\begin{tabular}{|c|c|c|c|c|c|c|c|c|}
\hline & Algorithm & No. of vectors & $W_{1}$ & $W_{2}$ & $W_{3}$ & $W_{4}$ & $W_{5}$ & Profile \\
\hline \multirow{12}{*}{ 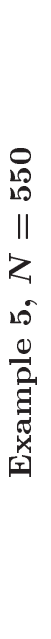 } & Sloan & & & & & & & 10530 \\
\hline & King & & & & & & & 10974 \\
\hline & PSO & $L=2$ & 0.0007 & 0.4852 & & & & 10501 \\
\hline & & $L=5$ & -0.0863 & 0.4638 & -0.3677 & 0.0034 & 0.9191 & 9280 \\
\hline & $\mathrm{CBO}$ & $L=2$ & 0.0043 & 0.4001 & & & & 10501 \\
\hline & & $L=5$ & 0.2191 & 0.9551 & -0.6962 & -0.0390 & -0.3207 & 9242 \\
\hline & ECBO & $L=2$ & 0.0001 & 0.9881 & & & & 10501 \\
\hline & & $L=5$ & -0.0255 & 0.8883 & -0.6256 & -0.0110 & -0.9183 & 9237 \\
\hline & TWO & $L=2$ & 0.0129 & 0.7645 & & & & 10501 \\
\hline & & $L=5$ & -0.0404 & 0.8801 & -0.5940 & -0.0063 & 0.7426 & 9237 \\
\hline & VPS & $L=2$ & 0.0024 & 0.4913 & & & & 10501 \\
\hline & & $L=5$ & -0.0917 & 0.9658 & -0.6458 & -0.0017 & 0.2137 & 9237 \\
\hline
\end{tabular}

Table 10. Comparison of the results of wavefront reduction for the shear wall problem.

\begin{tabular}{|c|c|c|c|c|c|c|c|c|}
\hline & Algorithm & No. of vectors & $W_{1}$ & $W_{2}$ & $W_{3}$ & $W_{4}$ & $W_{5}$ & $F_{r m s}$ \\
\hline \multirow{12}{*}{ 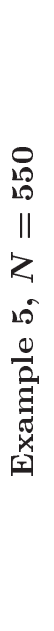 } & Sloan & & & & & & & 20.1739 \\
\hline & King & & & & & & & 21.0798 \\
\hline & $\mathrm{PSO}$ & $L=2$ & 0.3069 & 0.2202 & & & & 20.1401 \\
\hline & & $L=5$ & -0.3188 & 0.9852 & -0.7009 & 0.0489 & 0.118 & 17.2693 \\
\hline & $\mathrm{CBO}$ & $L=2$ & 0.8701 & 0.6144 & & & & 20.1401 \\
\hline & & $L=5$ & -0.1888 & 0.9093 & -0.8122 & 0.0210 & 0.8648 & 17.1544 \\
\hline & $\mathrm{ECBO}$ & $L=2$ & 0.8858 & 0.6517 & & & & 20.1401 \\
\hline & & $L=5$ & -0.1891 & -0.8720 & 0.9777 & 0.0199 & -0.4653 & 17.2239 \\
\hline & TWO & $L=2$ & 0.8711 & 0.6352 & & & & 20.1401 \\
\hline & & $L=5$ & 0.0101 & 0.9086 & -0.8164 & -0.0034 & -0.1742 & 17.1492 \\
\hline & VPS & $L=2$ & 0.2634 & 0.1834 & & & & 20.1401 \\
\hline & & $L=5$ & -0.2682 & -0.8807 & 0.8722 & -0.0001 & 0.5653 & 17.2057 \\
\hline
\end{tabular}

Table 11. Comparison of the results of profile reduction for the rectangular FEM with a four-opening problem.

\begin{tabular}{|c|c|c|c|c|c|c|c|c|}
\hline & Algorithm & No. of vectors & $W_{1}$ & $W_{2}$ & $W_{3}$ & $W_{4}$ & $W_{5}$ & Profile \\
\hline \multirow{12}{*}{ 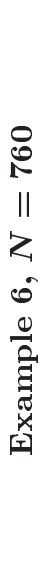 } & Sloan & & & & & & & 18719 \\
\hline & King & & & & & & & 18839 \\
\hline & PSO & $L=2$ & 0.6645 & 0.9066 & & & & 18690 \\
\hline & & $L=5$ & 0.1056 & 0.8858 & -0.4835 & -0.0152 & 0.0524 & 17136 \\
\hline & $\mathrm{CBO}$ & $L=2$ & 0.1899 & 0.2566 & & & & 18689 \\
\hline & & $L=5$ & -0.3332 & -0.7097 & 0.9412 & 0.0507 & 0.9747 & 17122 \\
\hline & $\mathrm{ECBO}$ & $L=2$ & 0.6665 & 0.9228 & & & & 18581 \\
\hline & & $L=5$ & -0.0458 & 0.7835 & -0.6332 & 0.0060 & -0.2254 & 17039 \\
\hline & TWO & $L=2$ & 0.7136 & 0.9633 & & & & 18581 \\
\hline & & $L=5$ & -0.0178 & -0.4092 & 0.9291 & 0.0024 & -0.5575 & 17039 \\
\hline & VPS & $L=2$ & 0.1785 & 0.2491 & & & & 18581 \\
\hline & & $L=5$ & -0.0513 & 0.796 & -0.3421 & 0.007 & 0.0739 & 17039 \\
\hline
\end{tabular}


Table 12. Comparison of the results of wavefront reduction for the rectangular with a four-opening problem.

\begin{tabular}{|c|c|c|c|c|c|c|c|c|}
\hline & Algorithm & No. of vectors & $W_{1}$ & $W_{2}$ & $W_{3}$ & $W_{4}$ & $W_{5}$ & $F_{r m s}$ \\
\hline \multirow{12}{*}{  } & Sloan & & & & & & & 25.9092 \\
\hline & King & & & & & & & 26.6508 \\
\hline & PSO & $L=2$ & 0.2053 & 0.3469 & & & & 25.8411 \\
\hline & & $L=5$ & -0.0879 & -0.3811 & 0.8933 & 0.012 & -0.1249 & 23.5891 \\
\hline & $\mathrm{CBO}$ & $L=2$ & 0.3181 & 0.5433 & & & & 25.8438 \\
\hline & & $L=5$ & -0.0713 & -0.6558 & 0.9556 & 0.0095 & -0.6861 & 23.4955 \\
\hline & $\mathrm{ECBO}$ & $L=2$ & 0.1855 & 0.3155 & & & & 25.8411 \\
\hline & & $L=5$ & -0.1056 & 0.8982 & -0.7698 & 0.0121 & -0.7255 & 23.4743 \\
\hline & TWO & $L=2$ & 0.5752 & 0.9624 & & & & 25.8438 \\
\hline & & $L=5$ & -0.0919 & 0.9571 & -0.6803 & 0.0130 & -0.0568 & 23.5090 \\
\hline & VPS & $L=2$ & 0.0287 & 0.05 & & & & 25.8438 \\
\hline & & $L=5$ & -0.0622 & -0.4264 & 0.9926 & 0.0086 & -0.2518 & 23.4599 \\
\hline
\end{tabular}

Table 13. Comparison of the results of profile reduction for the fan model.

\begin{tabular}{|c|c|c|c|c|c|c|c|c|}
\hline & Algorithm & No. of vectors & $W_{1}$ & $W_{2}$ & $W_{3}$ & $W_{4}$ & $W_{5}$ & Profile \\
\hline \multirow{12}{*}{ 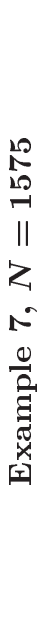 } & Sloan & & & & & & & 28703 \\
\hline & King & & & & & & & 28853 \\
\hline & PSO & $L=2$ & 0.2588 & 0.6068 & & & & 28629 \\
\hline & & $L=5$ & -0.2965 & 0.5407 & -0.6326 & -0.0214 & -0.1835 & 29674 \\
\hline & $\mathrm{CBO}$ & $L=2$ & 0.2129 & 0.4426 & & & & 28608 \\
\hline & & $L=5$ & -0.5700 & 0.8618 & -0.5831 & 0.0777 & 0.3363 & 27992 \\
\hline & $\mathrm{ECBO}$ & $L=2$ & 0.1765 & 0.9272 & & & & 28587 \\
\hline & & $L=5$ & -0.4186 & 0.9776 & -0.7792 & 0.1007 & -0.0557 & 27982 \\
\hline & TWO & $L=2$ & 0.1613 & 0.8465 & & & & 28579 \\
\hline & & $L=5$ & -0.3306 & 0.8144 & -0.5654 & 0.0668 & 0.6810 & 27977 \\
\hline & VPS & $L=2$ & 0.0274 & 0.1543 & & & & 28568 \\
\hline & & $L=5$ & -0.6613 & 0.8839 & -0.637 & 0.149 & -0.2288 & 27982 \\
\hline
\end{tabular}

Table 14. Comparison of the results of wavefront reduction for the fan model.

\begin{tabular}{|c|c|c|c|c|c|c|c|c|}
\hline & Algorithm & No. of vectors & $W_{1}$ & $W_{2}$ & $W_{3}$ & $W_{4}$ & $W_{5}$ & $F_{r m s}$ \\
\hline \multirow{12}{*}{ 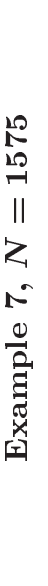 } & Sloan & & & & & & & 18.3958 \\
\hline & King & & & & & & & 18.4698 \\
\hline & PSO & $L=2$ & 0.0605 & 0.3289 & & & & 18.3126 \\
\hline & & $L=5$ & -0.0709 & 0.6589 & -0.9003 & -0.0677 & -0.4860 & 18.9964 \\
\hline & $\mathrm{CBO}$ & $L=2$ & 0.1382 & 0.8659 & & & & 18.3235 \\
\hline & & $L=5$ & -0.2174 & -0.4203 & 0.4590 & 0.0462 & -0.2162 & 19.2531 \\
\hline & $\mathrm{ECBO}$ & $L=2$ & 0.1688 & 0.8892 & & & & 18.3232 \\
\hline & & $L=5$ & 0.1883 & 0.7370 & -0.5928 & -0.0607 & -0.0945 & 18.6574 \\
\hline & TWO & $L=2$ & 0.0357 & 0.1982 & & & & 18.3240 \\
\hline & & $L=5$ & 0.0441 & 0.9101 & -0.7696 & 0.0139 & -0.6153 & 18.0211 \\
\hline & VPS & $L=2$ & 0.0597 & 0.3524 & & & & 18.3168 \\
\hline & & $L=5$ & -0.2561 & -0.3969 & 0.5031 & 0.0699 & -0.7877 & 18.2694 \\
\hline
\end{tabular}


Table 15. Comparison of the results of profile reduction for the H-shape problem.

\begin{tabular}{|c|c|c|c|c|c|c|c|c|}
\hline & Algorithm & No. of vectors & $W_{1}$ & $W_{2}$ & $W_{3}$ & $W_{4}$ & $W_{5}$ & Profile \\
\hline \multirow{12}{*}{ 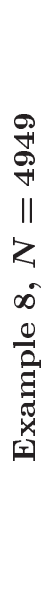 } & Sloan & & & & & & & 157457 \\
\hline & King & & & & & & & 157103 \\
\hline & PSO & $L=2$ & 0.0449 & 0.6963 & & & & 157095 \\
\hline & & $L=5$ & 0.1106 & 0.9323 & -0.0624 & -0.0284 & -0.3706 & 160705 \\
\hline & $\mathrm{CBO}$ & $L=2$ & 0.0229 & 0.9146 & & & & 157095 \\
\hline & & $L=5$ & -0.9709 & -0.9856 & 0.1853 & 0.2102 & -0.3565 & 159681 \\
\hline & $\mathrm{ECBO}$ & $L=2$ & 0.0620 & 0.9365 & & & & 157095 \\
\hline & & $L=5$ & -0.5805 & -0.7778 & 0.2437 & 0.1277 & -0.1065 & 159676 \\
\hline & TWO & $L=2$ & 0.0721 & 0.9800 & & & & 157095 \\
\hline & & $L=5$ & -0.8206 & -0.8691 & 0.5953 & 0.4801 & 0.2781 & 159675 \\
\hline & VPS & $L=2$ & 0.0081 & 0.8161 & & & & 157095 \\
\hline & & $L=5$ & -0.8685 & -0.9894 & 0.0888 & 0.1738 & -0.1293 & 159638 \\
\hline
\end{tabular}

Table 16. Comparison of the results of wavefront reduction for the H-shape problem.

\begin{tabular}{|c|c|c|c|c|c|c|c|c|}
\hline & Algorithm & No. of vectors & $W_{1}$ & $W_{2}$ & $W_{3}$ & $W_{4}$ & $W_{5}$ & $\boldsymbol{F}_{r m s}$ \\
\hline \multirow{12}{*}{ 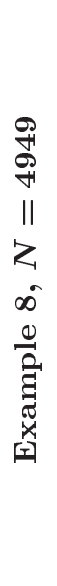 } & Sloan & & & & & & & 32.3665 \\
\hline & King & & & & & & & 32.2875 \\
\hline & PSO & $L=2$ & 0.0445 & 0.6963 & & & & 32.2869 \\
\hline & & $L=5$ & 0.2036 & -0.9340 & 0.0601 & 0.0151 & -0.5008 & 32.9486 \\
\hline & $\mathrm{CBO}$ & $L=2$ & 0.0361 & 0.8204 & & & & 32.2869 \\
\hline & & $L=5$ & -0.0469 & -0.9805 & 0.0890 & 0.0109 & -0.0406 & 32.7939 \\
\hline & $\mathrm{ECBO}$ & $L=2$ & 0.0145 & 0.6215 & & & & 32.2869 \\
\hline & & $L=5$ & -0.0665 & -0.9779 & 0.2937 & 0.0204 & 0.0726 & 32.8298 \\
\hline & TWO & $L=2$ & 0.0772 & 0.9898 & & & & 32.2869 \\
\hline & & $L=5$ & -0.3335 & -0.6943 & 0.6808 & 0.1610 & -0.0604 & 32.8845 \\
\hline & VPS & $L=2$ & 0.0025 & 0.3597 & & & & 32.2869 \\
\hline & & $L=5$ & -0.6995 & -0.8788 & 0.0746 & 0.1439 & 0.8584 & 32.7883 \\
\hline
\end{tabular}

In profile and wavefront reduction problems with $L=2$ and 5 methods, an attempt was made to display the applicability of using different priority functions by utilizing CBO, ECBO, TWO, and VPS algorithms. Optimal weights for these functions were achieved in the optimization processes for reducing the profile and wavefront of the stiffness matrices of the finite element models. According to Tables 1 to 16, it can be observed that Sloan and King's approaches can improve in most cases using some new parameters and weights. The weights obtained for different examples show that, compared to Sloan and King's algorithms, more suitable profile and wavefront values can be achieved by using the two-parameter method $(L=2)$. Compared to the two-parameter method and Sloan and King's algorithms, smaller profile and wavefront values can be attained in the five-parameter scheme $(L=5)$. However, for Example 8, the values of profile and wavefront of the Sloan and King's methods are smaller than those of the five-parameter algorithm.
It should be noted that, in $L=5$ approach, the active degrees are not updated as in Sloan's technique. Therefore, one should not always expect a better result when five adjusted parameters are employed instead of two free parameters. Since the graph properties employed in $L=5$ method are more than those in $L=2$ approach, the amount of reduction in profile and wavefront is higher than that in the Sloan and King's algorithms. The comparison results of the profile and wavefront reduction problem for Example 4 are shown in Figures 14 and 15, respectively.

The new recently developed metaheuristic algorithm, namely the vibrating particles system, has been employed in this study for the first time. Tables 1 to 16 demonstrate that this algorithm finds superior optimal values for profile and wavefront of most of the investigated problems. Moreover, the optimized results obtained by TWO and VPS can compete in performance with those obtained by the other three optimization methods of PSO, CBO, and ECBO and 


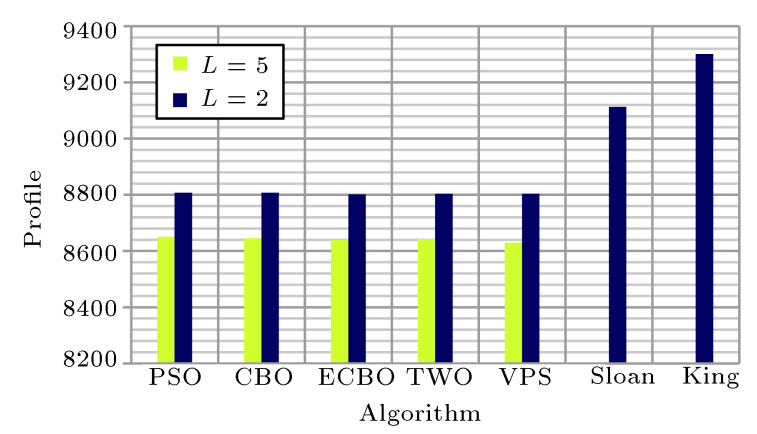

Figure 14. Comparison of profile results for Example 4.

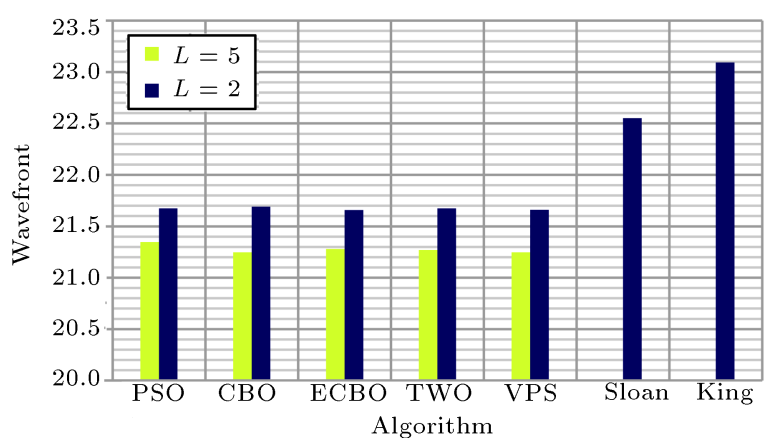

Figure 15. Comparison of the wavefront results for Example 4.

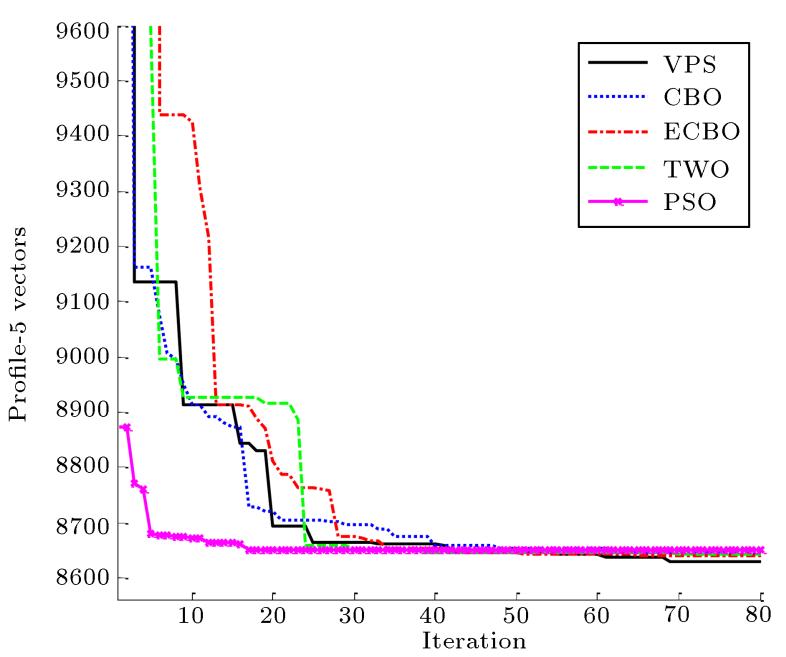

Figure 16. Convergence curves obtained for Example 4.

attain better results in some cases. Figures 16-17 and Figures 18-19 show the convergence rate comparisons of these algorithms for profile and wavefront reduction in the case of Examples 4 and 6, respectively.

It should be mentioned that the optimization proposed in this paper was performed to improve the coefficients of Sloan's algorithm and was not necessarily limited to the profile reduction of sparse matrices. A direct optimization may or may not result in better values for the profile of the models.

Although the methods of this paper are used for nodal ordering in the stiffness method, their application

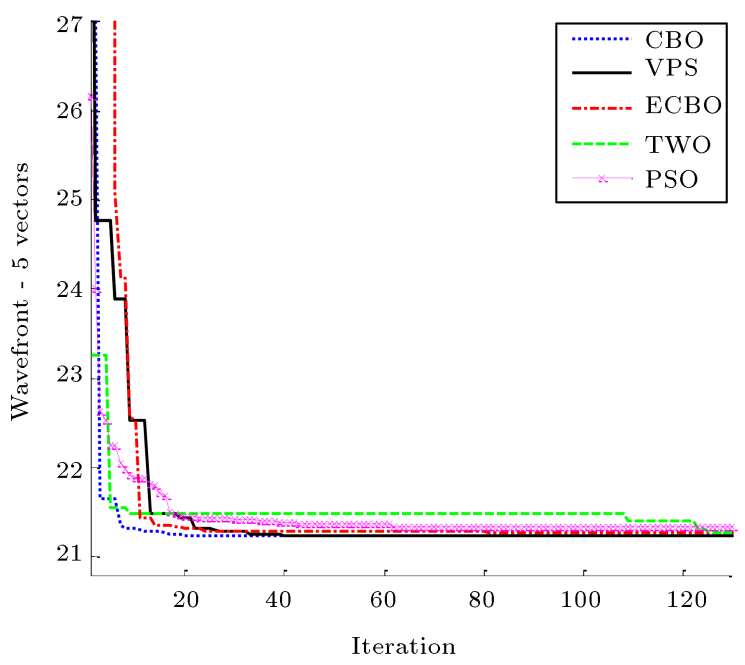

Figure 17. Convergence curves obtained for Example 4.

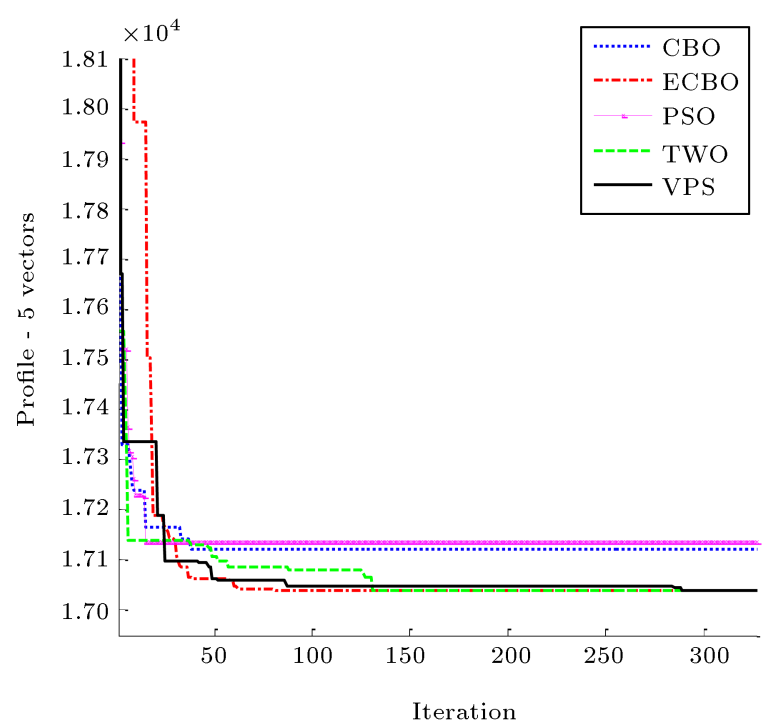

Figure 18. Convergence curves obtained for Example 6 .

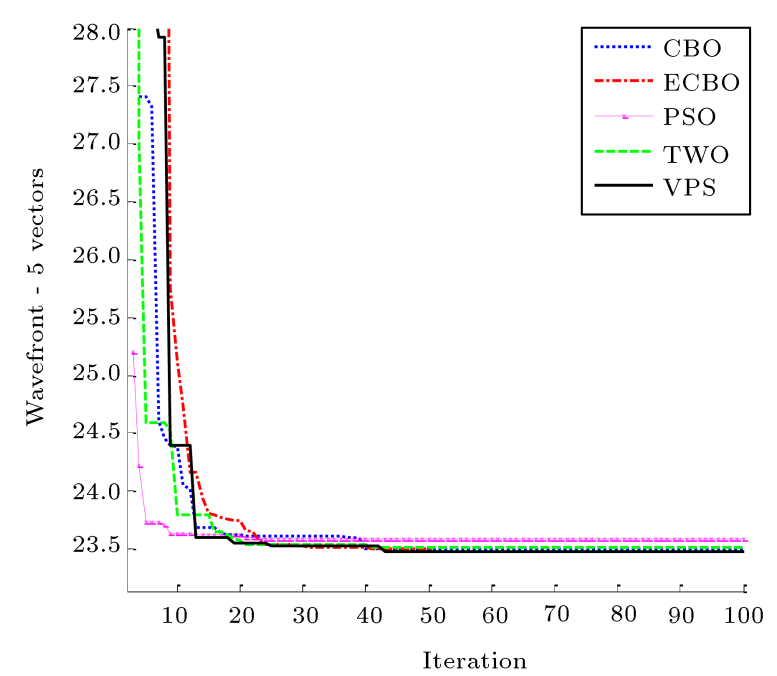

Figure 19. Convergence curves obtained for Example 6. 
can easily be extended to ordering the self-equilibrating systems in the force method.

\section{References}

1. Kaveh, A. "Applications of topology and matroid theory to the analysis of structures", Ph.D. Thesis, Imperial College of Science and Technology, London University, UK (1974).

2. Kaveh, A., Structural Mechanics: Graph and Matrix Methods, Research Studies Press, 3rd edition, Somerset, UK (2004).

3. Kaveh, A., Optimal Structural Analysis, John Wiley, 2nd Edn., Chichester, UK (2006).

4. Papademetrious, C.H. "The NP-completeness of bandwidth minimization problem", Comput. J., 16, pp. 177-192 (1976).

5. Gibbs, N.E., Poole, W.G., and Stockmeyer, P.K. "An algorithm for reducing the bandwidth and profile of a sparse matrix", SIAM J. Numer. Anal., 12, pp. 236250 (1976).

6. Cuthill, E. and McKee, J. "Reducing the bandwidth of sparse symmetric matrices", Proceedings of the 24 th National Conference ACM, Bradon System Press, NJ, pp. 157-172 (1969).

7. Bernardes, J.A.B. and Oliveira, S.L.G.D. "A systematic review of heuristics for profile reduction of symmetric matrices", Procd. Comput. Sci., 51, pp. 221-230 (2015).

8. King, I.P. "An automatic reordering scheme for simultaneous equations derived from network systems", Int. J. Numer. Methods Eng., 2, pp. 523-533 (1970).

9. Kaveh, A. and Behzadi, A.M. "An efficient algorithm for nodal ordering of networks", Iran. J. Sci. Technol., Transactions in Civil Engineering, 11, pp. 11-18 (1987).

10. Kaveh, A. and Roosta, G.R. "Comparative study of finite element nodal ordering methods", Eng. J., 20(1\&2), pp. 86-96 (1998).

11. Koohestani, B. and Poli, R. "Addressing the envelope reduction of sparse matrices using a genetic programming system", Comput. Optimiz. Appl., 60, pp. 789814 (2014).

12. Kaveh, A., Advances in Metaheuristic Algorithms for Optimal Design of Structures, 2nd Edn., Springer International Publishing, Switzerland (2017).

13. Kaveh, A. and Mahdavi, V.R. "Colliding bodies optimization: A novel meta-heuristic method", Comput. Struct., 139, pp. 18-27 (2014).

14. Kaveh, A. and Ilchi Ghazaan, M. "Enhanced colliding bodies optimization for design problems with continuous and discrete variables", Adv. Eng. Softw., 77, pp. 66-75 (2014).

15. Kaveh, A. and Zolghadr, A. "A novel meta-heuristic algorithm: tug of war optimization", Int. J. Optim. Civil Eng., 6, pp. 469-492 (2016).
16. Kaveh, A. and Ilchi Ghazaan, M. "A new metaheuristic algorithm: vibrating particles system", Sci. Iran., 24(2), pp. 551-566 (2017).

17. Sloan, S.W. "An algorithm for profile and wavefront reduction of sparse matrices", Int. J. Numer. Methods Eng., 23, pp. 1693-1704 (1986).

18. Kaveh, A. and Rahimi Bondarabdy, H.A. "A hybrid method for finite element ordering", Comput. Struct., 80(3-4), pp. 219-225 (2002).

19. Rahimi Bondarabady, H.A., and Kaveh, A. "Nodal ordering using graph theory and a genetic algorithm", Finite Elem. Anal. Des., 40(9-10), pp. 1271-1280 (2004).

20. Kaveh, A. and Sharafi, P. "Optimal priority functions for profile reduction using ant colony optimization", Finite Elem. Anal. Des., 44, pp. 131-138 (2008).

21. Kaveh, A. and Sharafi, P. "Ordering for bandwidth and profile minimization problems via charged system search algorithm", Iran. J. Sci. Technol., Transactions in Civil Engineering, 36(C1), pp. 39-52 (2012).

22. Kaveh, A. and Bijari, Sh. "Bandwidth, profile and wavefront optimization using PSO, CBO, ECBO and TWO algorithms", Iran. J. Sci. Technol., Transactions in Civil Engineering, 41(1), pp. 1-12 (2017).

23. Everstine, G.C. "A comparison of three resequencing algorithms for the reduction of matrix profile and wavefront", Int. J. Numer. Methods Eng., 14(6), pp. 837-853 (1979).

\section{Biographies}

Ali Kaveh was born in 1948 in Tabriz, Iran. After graduation from the Department of Civil Engineering at the University of Tabriz in 1969, he continued his studies on Structures at Imperial College of Science and Technology at London University and received his MSc, DIC, and PhD degrees in 1970 and 1974, respectively. He then joined the Iran University of Science and Technology. Professor Kaveh is the author of 625 papers published in international journals and 155 papers presented at national and international conferences. He has authored 23 books in Farsi and 8 books in English published by Wiley, RSP, American Mechanical Society, and Springer.

Shima Bijari is a $\mathrm{PhD}$ candidate in Civil Engineering Department of Iran University of Science and Technology. She took a BSc degree in 2012 and MSc in 2014. Her main expertise and experience reside in the field of structural engineering and optimal design of structures. She is interested in metaheuristic algorithms and their applications. 